\title{
Correlation Lengths of Linear and Branched Polymers in a Good Solvent
}

\author{
Satoshi YukioKA, ${ }^{* 1}$ Yuji Higo, ${ }^{* 2}$ Ichiro Noda, \\ and Mitsuru NAGASAWA \\ Department of Synthetic Chemistry, Nagoya University, \\ Furo-cho, Chikusa-ku, Nagoya 464, Japan
}

(Received April 30, 1986)

\begin{abstract}
Small angle X-ray scattering and light scattering from linear and branched polymers in a good solvent were measured to study the concentration dependence of correlation length $\xi$ over a wide range of concentration and the effects of branching on $\xi$. The concentration dependence of $\xi$ of linear polymers in semidilute solutions is well explained by the scaling theory and the reduced correlation length $\xi /\left\langle s^{2}\right\rangle^{1 / 2}$ of linear polymers is found to be an universal function of the degree of coil-overlapping $C / C^{*}$ over wide ranges of molecular weight and concentration in semidilute solutions as predicted by the scaling theory. The effects of branching are found to be not so remarkable that $\xi$ of branched polymers is almost equal to that of linear polymers in the same concentration.
\end{abstract}

KEY WORDS Correlation Length / Semidilute Solution / Linear Polymer / Branched Polymer / Good Solvent / Small Angle X-Ray Scattering / Light Scattering / Scaling Theory /

From extensive studies of osmotic pressure of linear polymer solutions ${ }^{1-3}$ it was pointed out that polymer solutions in good solvent systems can be classified into the three different regions, that is, dilute $(C<1$ and $\left.C / C^{*}<1\right)$, semidilute $\left(C<1\right.$ but $\left.C / C^{*}>1\right)$ and concentrated solutions $(\mathrm{C} \sim 1$ and $C /$ $\left.C^{*}>1\right)$ if we employ two concentration parameters $C$ and $C / C^{*}$ to specify polymer solutions, where $C$ is the polymer concentration and $C / C^{*}$ is the degree of coil-overlapping. Here, $C^{*}$ is the critical concentration at which the polymer coils begin to overlap with each other as defined by

$$
C^{*}=3 M /\left(4 \pi\left\langle s^{2}\right\rangle^{3 / 2} N_{\mathrm{A}}\right)
$$

where $M$ and $\left\langle s^{2}\right\rangle$ are the molecular weight and mean square radius of gyration of the polymer at the infinite dilution, respectively and $N_{\mathrm{A}}$ is the Avogadro's number.

It was also shown ${ }^{1-3}$ that the reduced osmotic pressure $\Pi M / C R T$ of linear polymers in semidilute solutions can be expressed as an universal function of $C / C^{*}$ and is well explained by the scaling theory ${ }^{4-6}$ such as

$$
\Pi M / C R T \propto\left(C / C^{*}\right)^{1 /(3 v-1)}
$$

and

$$
\Pi / R T \propto C^{3 v /(3 v-1)}
$$

where $\Pi$ is the osmotic pressure, $R$ is the gas constant and $v$ is the excluded volume exponent in the relationship between $\left\langle s^{2}\right\rangle$ and $M$, i.e., $\left\langle s^{2}\right\rangle \propto M^{2 v}$.

As shown in a previous paper ${ }^{7}$ the scaling

Present address: *1 Polymer Research Laboratory, Toyo Soda Manufacturing Co., Ltd., 1-8 Kasumi, Yokkaichi, Mie 510; *2 Tokyo Research Center, Toyo Soda Manufacturing Co., Ltd., 2743-1 Hayakawa, Ayase, Kanagawa 252. 


\section{S. YUKIOKA et al.}

theory, eq 2 is also valid for osmotic pressure of semidilute solutions of star-shaped branched polymers with three arms in good solvents, while it is valid for that of highly branched polymers in the concentration range where polymer coils extensively overlap with each other. In these causes the concentration dependence of $\Pi$ are quantitatively explained by eq 2 assuming that the values of $v$ for the branched polymers are equal to those for the corresponding linear polymers in good solvents. The deviation from the scaling theory of linear polymers observed for highly branched polymer at a relatively low degree of coil-overlapping in semidilute solutions may be explained by assuming that there is a region from which segments of other molecules are excluded as a result of high segment density as pointed out by Daoud and Cotton. ${ }^{8}$

According to the scaling theory ${ }^{5,6}$ the correlation length $\xi$ of linear polymer, which is a characteristic length in semidilute solutions is also given by

$$
\xi /\left\langle s^{2}\right\rangle^{1 / 2} \propto\left(C / C^{*}\right)^{-v /(3 v-1)}
$$

and

$$
\xi \propto C^{-v /(3 v-1)}
$$

Although several experimental works ${ }^{5,9,10}$ were carried out so far on the correlation length of polymer in good solvents, the ranges of polymer concentration $C$ and degree of coil-overlapping $C / C^{*}$ are not wide enough to examine the scaling theory. Since the branching affects the concentration dependence of $\Pi$ in semidilute solutions of highly branched polymers as described above, ${ }^{7}$ it is interesting to study the effects of branching on the scattering function and correlation length $\xi$. In this work, therefore, we measured the correlation lengths of linear polystyrenes and $\operatorname{poly}(\alpha-$ methylstyrene)s with narrow molecular weight distributions over a wide range of molecular weight and of branched polystyrenes with many long branches by using small angle X- ray scattering and light scattering methods. Here, we report that the concentration dependence of $\xi$ of linear polymers in semidilute solutions is well explained by the scaling theory and the reduced correlation length $\xi /$ $\left\langle s^{2}\right\rangle^{1 / 2}$ of linear polymers is found to be an universal function of the degree of coiloverlapping $C / C^{*}$ over wide range of molecular weight and concentration as predicted by the scaling theory. Moreover, we report that the effects of branching are not so remarkable that $\xi$ of branched polymers is almost equal to that of linear polymers at the same concentration.

\section{EXPERIMENTAL}

\section{Materials}

Linear polystyrenes with narrow molecular weight distributions were purchased from Pressure Chemical Co., Ltd. (PS-16, PS-90 and PS-180) and Toyo Soda Manufacturing Co., Ltd. (F-450, F-850 and F-2000). Poly$(\alpha$-methylstyrene)s with narrow molecular weight distributions $(\alpha-113$ and $\alpha-111)$ were prepared previously by an anionic polymerization method. ${ }^{11}$ Branched polystyrenes (KIII-F89, KI-F3 and K0-F3) were also prepared previously by an anionic polymerization method. KIII-F89 and KI-F3 have

Table I. Molecular characteristics of linear polymers

\begin{tabular}{cccc}
$\begin{array}{c}\text { Sample } \\
\text { code }\end{array}$ & $\bar{M}_{w} \times 10^{-4}$ & $\begin{array}{c}\left\langle S^{2}\right\rangle \times 10^{11} / \\
\mathrm{cm}^{2 \mathrm{a}}\end{array}$ & $\begin{array}{c}C^{*} \times 10^{2} / \\
\mathrm{g} \mathrm{cm}^{-3 \mathrm{~b}}\end{array}$ \\
\hline $\begin{array}{c}\text { Polystyrenes } \\
\text { PS-16 }\end{array}$ & 15.7 & $0.02_{1}$ & $2.0_{4}$ \\
PS-90 & 90.1 & $1.8_{6}$ & 0.52 \\
PS-180 & 193 & $4.1_{7}$ & $0.28_{4}$ \\
F-450 & 448 & $11_{3}$ & $0.15_{4}$ \\
F-850 & 842 & $21_{3}$ & 0.090 \\
F-200 & 2100 & $70_{.0}$ & 0.045 \\
Poly $(\alpha-$ methylstyrene)s & & \\
$\alpha-113$ & 330 & $7.3_{3}$ & $0.20_{9}$ \\
$\alpha-111$ & 747 & 18.7 & $0.11_{6}$ \\
\hline
\end{tabular}

${ }^{a}$ Evaluated from $\left\langle S^{2}\right\rangle=1.38 \times 10^{-18} M^{1.19}$ for polystyrenes in toluene at $25^{\circ} \mathrm{C}^{7}$

${ }^{b}$ In toluene at $25^{\circ} \mathrm{C}$. 
Table II. Molecular characteristics of branched polymers

\begin{tabular}{|c|c|c|c|c|}
\hline $\begin{array}{l}\text { Sample } \\
\text { code }\end{array}$ & $\bar{M}_{w} \times 10^{-4}$ & $f^{b}$ & $\begin{array}{c}\left\langle S^{2}\right\rangle \times 10^{11} / \\
\mathrm{cm}^{2 \mathrm{c}}\end{array}$ & $\begin{array}{l}C^{*} \times 10^{2} / \\
\mathrm{gcm}^{-3 \mathrm{c}}\end{array}$ \\
\hline KIII-F89a & 265 & 15 & $2.3_{5}$ & $0.92_{2}$ \\
\hline $\mathrm{KI}-\mathrm{F}^{\mathrm{a}}$ & 96 & 16 & 0.85 & $1.5_{4}$ \\
\hline $\mathrm{K} 0-\mathrm{F} 3^{\mathrm{a}}$ & 358 & 52 & $1.2_{9}$ & $3.0_{5}$ \\
\hline
\end{tabular}

a The number-average molecular weights of a backbone $\left(\bar{M}_{n \mathrm{o}}\right)$ and branch $\left(\bar{M}_{n \mathrm{~b}}\right)$ are $9.8 \times 10^{4}$ and $17.6 \times 10^{4}$ for KIII-F89, $5.7 \times 10^{4}$ and $5.5 \times 10^{4}$ for KI-F3, and $9.5 \times 10^{4}$ and $6.5 \times 10^{4}$ for K0-F3, respectively.

b $f=\left(\bar{M}_{w}-\bar{M}_{n \mathrm{o}}\right) / \bar{M}_{n \mathrm{~b}}$.

c In toluene at $25^{\circ} \mathrm{C}$.

$15 \sim 16$ branches while K0-F3 has 52 branches, all long enough compared with the parent polymers. $^{7,12}$ Thus, they may be regarded as star-shaped polymers with many branches. The molecular characteristics for those linear and branched polymers are shown in Tables I and II, respectively.

Toluene, which is a good solvent for polystyrenes and poly( $\alpha$-methylstyrene)s was purified by the same method as described in the previous paper. ${ }^{11}$

\section{Measurements}

Small Angle X-Ray Scattering. The measurements were carried out with a Kratky U-slit camera of Anton Paar Co. with a step scanner at $25^{\circ} \mathrm{C}$. The $\mathrm{X}$-ray source was a water cooled copper-anode tube operated at $45 \mathrm{kV} \times 30$ $\mathrm{mA}$ powered by a X-ray generator, Model DX-GE-2D of JEOL (Japan Electron Optic Laboratory Co.). The wave length was 1.54 $\AA\left(\mathrm{Cu}-K_{\alpha}\right)$. Stability of the beam intensity was confirmed by using Luporene in each measurement. The widths of entrance and counter slits were 100 and $250 \mu \mathrm{m}$, respectively and the distance between the sample position and the plane of resistration was $21 \mathrm{~cm}$. The difference between the scattering intensities from solution and solvent was converted to desmeared intensities $I(q)$ according to the method of Glatter for a slit correction as described previously. ${ }^{13}$
Light Scattering. The measurements were carried out with a Fica 50 automatic light scattering photometer at $25^{\circ} \mathrm{C}$. The incident beam was an unpolarized light with a wave length of $436 \mathrm{~nm}$ and the scattering angle was in the range of $30 \sim 150^{\circ}$. The optical constant was determined with the scattering intensity from benzene at $25^{\circ} \mathrm{C}$. The optical purification of solutions were usually carried out by filtration through $0.45 \mu \mathrm{m}$ Millipore membrane filters. Solutions of high molecular weight samples were clarified by ultracentrifugation at $50,000 \mathrm{~g}$ for $c a .2 \mathrm{~h}$.

Concentration ranges for measurement were $0.02 \sim 0.3 \mathrm{~g} \mathrm{~cm}^{-3}$ in small angle $\mathrm{X}$-ray scattering and $0.003 \sim 0.01 \mathrm{~g} \mathrm{~cm}^{-3}$ in light scattering, respectively.

\section{RESULTS}

The correlation length $\xi$ in semidilute solutions is usually evaluated by using the following Lorentzian equation from angular dependence of scattering intensity $I(q)$ in the range of $1 /\left\langle s^{2}\right\rangle^{1 / 2}<q<1 / a$. $^{6}$

$$
I(q) \propto 1 /\left(1+q^{2} \xi^{2}\right)
$$

where $q$ is the magnitude of the wave vector $q=(4 \pi / \lambda) \sin (\theta / 2), \lambda$ is the wave length in the solvent, $\theta$ is the scattering angle and $a$ is the statistical segment length of the polymer.

It should be noted, however, that eq 4 is rigorous when the mean field approximation is valid, for instance, in concentrated solutions as shown by Edwards, ${ }^{14}$ Jannink and de Gennes. ${ }^{15}$ Benoit and Benmouna ${ }^{16}$ also showed that eq 4 can be considered as a direct application of the equation of Ornstein and Zernike. Moreover, Daoud et al. ${ }^{5}$ assumed that eq 4 is still valid in semidilute solutions if a chain behaves like a Gaussian chain whose statistical segment length is $\xi$ and the renomalized excluded volume is used.

The validity of eq 4 in semidilute solutions, therefore, should be examined by experiments. Daoud et al. $^{5}$ reported that there is a good 


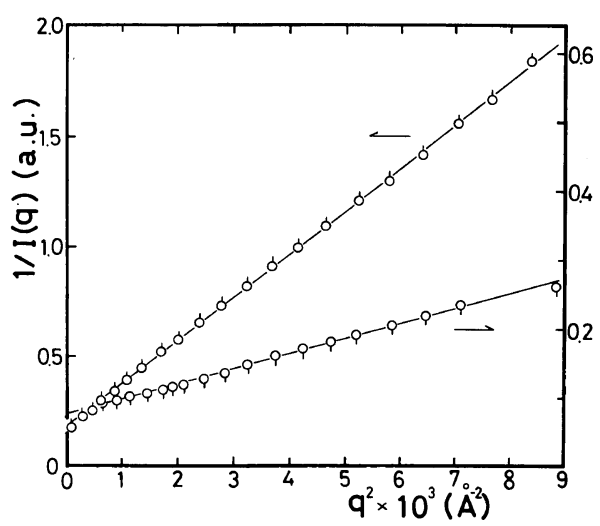

Figure 1. Plots of invèrse of small angle X-ray scattering intensity against $q^{2}$ for linear polystyrenes in toluene at $25^{\circ} \mathrm{C}$. The symbols $Q$ and $O$ denote the data for PS$90\left(C=0.101 \mathrm{~g} \mathrm{~cm}^{-3}\right)$ and $\mathrm{F}-450 \quad\left(C=0.029 \mathrm{~g} \mathrm{~cm}^{-3}\right)$, respectively.

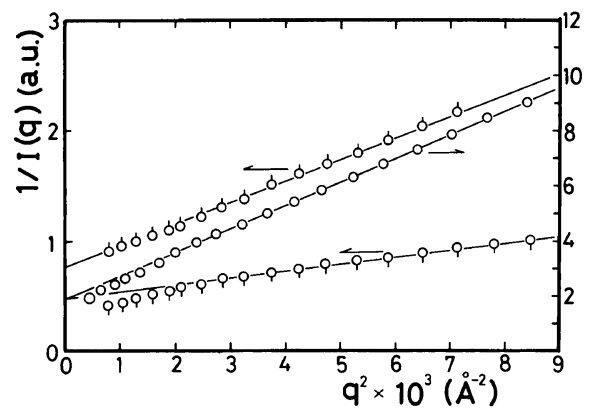

Figure 2. Plots of inverse of small angle $X$-ray scattering intensity against $q^{2}$ for branched polystyrenes in toluene at $25^{\circ} \mathrm{C}$. The symbols $\mathrm{O}, \phi$, and $\bigcirc$ denote the data for KIII-F89 $\left(C=0.205 \mathrm{~g} \mathrm{~cm}^{-3}\right)$, KI-F3 $(C=$ $\left.0.119 \mathrm{~g} \mathrm{~cm}^{-3}\right)$ and $\mathrm{K} 0-\mathrm{F} 3\left(C=0.052 \mathrm{~g} \mathrm{~cm}^{-3}\right)$, respectively.

linear relationship between $1 / I(q)$ and $q^{2}$ in small angle neutron scattering from deuterated polystyrenes in $\mathrm{CS}_{2}$ as predicted by eq 4 . As shown in Figures 1 and 2 the same good linear relationship was observed except for low $q$ regions in small angle $\mathrm{X}$-ray scattering from linear and branched polystyrenes in toluene. The scattering data at the low $q$ regions were neglected to evaluate $\xi$ since the desmeared intensities at the low $q$ regions were very much affected by the desmearing conditions.

In light scattering data the deviation from the linearity was also observed at low $q$ regions

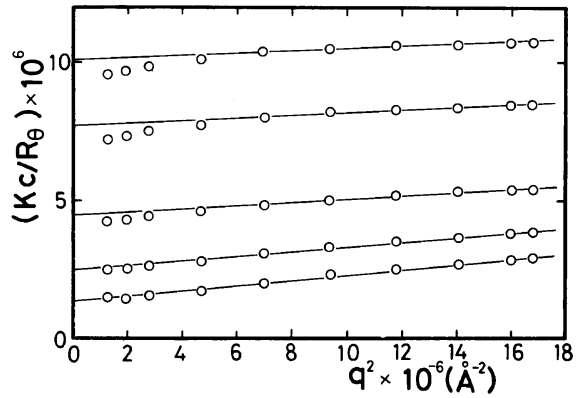

Figure 3. Plots of inverse of light scattering intensity against $q^{2}$ for the linear polystyrene F-450 in toluene at $25^{\circ} \mathrm{C}$. The concentrations are $0.0099,0.0080,0.0051$, 0.0031 , and $0.0018 \mathrm{~g} \mathrm{~cm}^{-3}$ from top to bottom.

where $q^{2}\left\langle s^{2}\right\rangle<1$, when the polymer concentrations becomes higher as shown in Figure 3. It was pointed out ${ }^{17}$ that the deviation arises from the presence of large concentration fluctuations with correlation lengths comparable to the radius of gyration. In the present work, therefore, $\xi$ was evaluated from angular dependence of light scattering at the high $q$ regions where $q^{2}\left\langle s^{2}\right\rangle>1$ according to eq 4 as shown in Figure 3.

\section{DISCUSSION}

Double logarithmic plots of $\xi$ of linear polymers determined as described above against $\mathrm{C}$ are shown in Figure 4. The data in range of $C>0.01 \mathrm{~g} \mathrm{~cm}^{-3}$ were obtained by small angle $\mathrm{X}$-ray scattering, while those in range of $C<0.01 \mathrm{~g} \mathrm{~cm}^{-3}$ by light scattering. Data of neutron scattering from deuterated polystyrenes in $\mathrm{CS}_{2}{ }^{5}$ are also included. The polymer concentration dependence of correlation length $\xi$ is independent of the methods of measurement. And also no difference is found between polystyrene and poly $(\alpha$-methylstyrene). The solid line has the slope of -0.75 as predicted from the scaling theory, ${ }^{5,6}$ eq $3 \mathrm{~b}$, assuming that $v=0.6$. The same data were replotted in the form of $\xi /\left\langle s^{2}\right\rangle^{1 / 2} v s$. $C / C^{*}$ in Figure 5. The solid line again has the slope of -0.75 . The reduced correlation length $\xi /\left\langle s^{2}\right\rangle^{1 / 2}$ for linear polymers is found to be an 


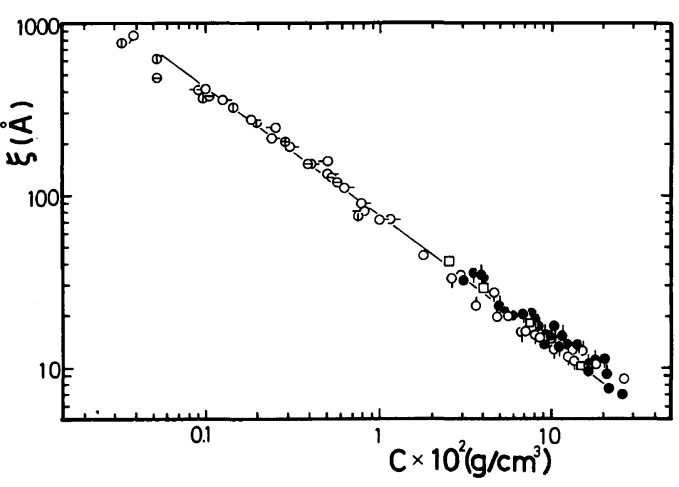

Figure 4. Concentration dependence of correlation lengths $\xi$ of linear and branched polymers in good solvents. The symbols are as follows: linear polystyrenes in toluene ( $O$, PS-16; $\bigcirc$, PS-90; Q, PS-180; O-, F-450; $-\bigcirc, \mathrm{F}-850$; $\bigcirc, \mathrm{F}-2000)$; linear poly( $\alpha$-methylstyrene)s in toluene (1), $\alpha-113 ; \ominus, \alpha-111)$; linear deuterated polystyrene in $\mathrm{CS}_{2}(\square)^{5}$; branched polystyrenes in toluene (Q, KIII-F89; $\mathbf{O}$, KI-F3; $\mathbf{O}, \mathrm{K} 0-\mathrm{F} 3$ ). The line denotes eq $3 \mathrm{~b}$ with $v=0.6$.

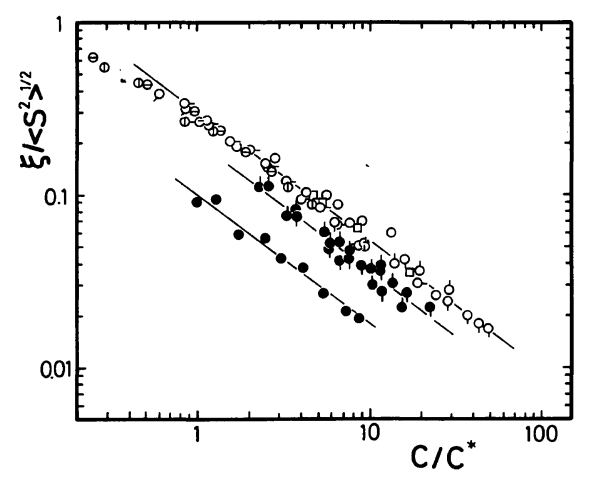

Figure 5. Double logarithmic plots of reduced correlation lengths against $C / C^{*}$. The symbols are the same as in Figure 4. The line denotes eq 3 a with $v=0.6$.

universal function of $C / C^{*}$ over wide range of molecular weight and concentration. Thus, when the polymer concentration $C$ is higher than the critical concentration $C^{*}$ and lower than a certain concentration $C^{* *} \sim 0.2 \mathrm{~g} \mathrm{~cm}^{-3}$, the prediction of the scaling theory is well supported by experiments, in agreement with the conclusion from osmotic pressure. ${ }^{1-3}$

If there is a region in which segments of other chains cannot penetrate in highly branched polymers as suggested previously, we may have two characteristic lengths, that is, a correlation length similar to that found in semidilute solutions of linear polymer and a radius inside which the segments of other chains do not penetrate, as pointed out by Daoud and Cotton. ${ }^{8}$ However, we cannot observe any particular difference between the scattering functions of linear and branched polymers within experimental errors as shown in Figures 1 and 2. So we evaluated the correlation lengths of branched polymers by using eq 4 .

Figure 4 shows that the correlation lengths of branched polymers are almost equal to but may be slightly larger than those of linear polymers at the same polymer concentration. This result appears to be consistent with the fact that the osmotic pressure of branched polymers ${ }^{7}$ is lower than that of linear polymers except for a region where polymer coils extensively overlap, considering that $\Pi \sim 1 / \xi^{3}{ }^{6}$. Since the concentration dependence of $\xi$ is weaker than that of $\Pi$, however, it is difficult to conclude that there is a significant difference between the correlation lengths of linear and branched polymers as observed for $\Pi,{ }^{7}$ considering the experimental errors in determining $\xi$. If the reduced correlation lengths of branched polymers are compared with those of linear polymers, data of branched polymers are lower than those of linear polymers as shown in Figure 5. This is a natural result obtained from the fact that the segment density of branched polymer is higher than that of linear polymer having the same molecular weight.

\section{REFERENCES}

1. I. Noda, N. Kato, T. Kitano, and M. Nagasawa, Macromolecules, 14, 668 (1981).

2. J. des Cloizeaux and I. Noda, Macromolecules, 15, 1505 (1982).

3. I. Noda, Y. Higo, N. Ueno, and T. Fujimoto, Macromolecules, 17, 1055 (1984).

4. J. des Cloizeaux, J. Phys. (Paris), 36, 281 (1975).

5. M. Daoud, J. P. Cotton, B. Farnoux, G. Jannink, G. Sama, H. Benoit, C. Dupplessix, C. Picot, and P. G. 


\section{S. YuKIOKa et al.}

de Gennes, Macromolecules, 8, 804 (1975).

6. P. G. de Gennes, "Scaling Concepts in Polymer Physics," Cornell University Press, Ithaca, N. Y., 1980.

7. Y. Higo, N. Ueno, and I. Noda, Polym. J., 15, 367 (1983).

8. M. Daoud and J. P. Cotton, J. Phys. (Paris), 43, 531 (1982).

9. P. Wiltzius, H. R. Haller, D. S. Cannell, and D. W. Sheafer, Phys. Rev. Lett., 51, 1183 (1983).

10. F. Hamada, S. Kinugasa, M. Hayashi, and A. Nakajima, Macromolecules, 18, 2290 (1985).

11. T. Kato, K. Miyaso, I. Noda, T. Fujimoto, and M.
Nagasawa, Macromolecules, 3, 777 (1970).

12. I. Noda, T. Horikawa, T. Kato, T. Fujimoto, and M. Nagasawa, Macromolecules, 3, 795 (1970).

13. Y. Muroga, I. Sakuragi, I. Noda, and M. Nagasawa, Macromolecules, 17, 1844 (1984).

14. S. F. Edwards, Proc. R. Soc. London, Ser. A, 88, 265 (1966).

15. G. Jannink and P. G. de Gennes, J. Chem. Phys., 48, 225 (1968).

16. H. Benoit and M. Benmouna, Polymer, 25, 1059 (1984).

17. J. T. Koberstein, C. Picot, and H. Benoit, Polymer, 26, 673 (1985). 